\title{
Effects of Joint Mobilization Versus Acupuncture on Pain and Functional Ability in People with Chronic Neck Pain: A Randomized Controlled Trial of Comparative Effectiveness
}

\author{
Petros Voulgarakis, Paris lakovidis, Dimitrios Lytras*, loanna P. Chatziprodromidou, \\ Anastasios Kottaras, Thomas Apostolou
}

Department of Physiotherapy, Faculty of Health Sciences, International Hellenic University, Thessaloniki, Greece

Received May 25, 2021

Revised October 30, 2021

Accepted November 15, 2021

Correspondence to

Dimitrios Lytras

Department of Physiotherapy, Faculty

of Health Sciences, International

Hellenic University, Thessaloniki,

Greece

E-mail lytrasde@gmail.com
Background: Chronic Neck Pain (CNP) is one of the main contributing factors to disability in people of working age.

Objectives: The aim of this randomized clinical trial was to compare the efficacy between acupuncture and joint mobilization on pain and disability in patients with CNP.

Methods: The study involved 45 men and women with CNP, divided into three groups of 15 each. The first group followed a manual therapy protocol with joint mobilization for eight weeks three times per week. The second group followed an acupuncture protocol of equal duration and frequency, while the third group did not follow any treatment. Pain with the Visual Analog Scale (VAS) for pain and functional limitations with the Neck Disability Index (NDI) questionnaire were assessed before and after an 8-week intervention. Analysis of variance was applied while post-hoc comparisons were made to determine the differences among the groups at each time of measurement.

Results: Both intervention groups showed statistically significant differences compared to the control group after the end of the intervention in both the VAS and the NDI scores $(p<$ .001). Furthermore, the acupuncture group showed a statistically significant improvement compared to the joint mobilization team after the end of the intervention in the VAS score ( $p$ $<.001$ ) and the NDI score $(p<.05)$.

Conclusion: Both joint mobilization and acupuncture appear to be effective interventions in reducing pain and improving functional ability in people with CNP. However, acupuncture appears to have a greater analgesic effect than joint mobilization.

Keywords: Chronic neck pain, Acupuncture, Joint mobilization, Rehabilitation

\section{INTRODUCTION}

Chronic neck pain is defined as neck pain with symptoms that manifest for more than three months [1,2]. Millions of people worldwide are directly affected by chronic neck pain both personally and socially [3-6] According to epidemiological data in the USA alone, the costs associated with treating patients with chronic neck pain increased by $65 \%$ between 1997 and $2005.50 \%$ of episodes of acute neck pain will continue to show symptoms for a long time $[7,8]$. Nonspecific neck pain is defined as neck pain whose presence is not associated with any serious pathology and can be managed through various therapeutic regimens such as pharmacotherapy, acupuncture, physiotherapy and exercise

\section{$[2,3,5,9-13]$}

Manual techniques as well as complementary therapies have been shown to help treat chronic neck pain effectively $[10,14-18]$. Two of the methods that have been widely used in clinical practice in recent decades are joint mobilization and acupuncture $[2,10,19]$.

Joint mobilization is a manual technique that is comprised from specialized continuous passive movements on the joints. These movements, according to the International Federation of Orthopedic Manipulative Physical Therapists (IFOMPT) [20] are varied in speed and range. Possibly they may be shortrange and high-speed manipulations with the aim to restore optimal movement, functioning and reduce pain.

Joint mobilization is part of manual therapy, which is 
defined as the set of dexterous movements applied with the hands, with the aim of direct or indirect relief of the patient from pain by the World Confederation for Physical Therapy [21].

Research data from recent decades have shown that the application of joint mobilization significantly reduces pain and improves the functioning of patients with chronic neck pain [22-24]. However, the findings of the studies show great heterogeneity in terms of their results $[2,22,25]$. This may be due to the heterogeneity observed between the different protocols in terms of time, duration, dosage and the appropriate combination of techniques used [9,22]. Tsakitzidis et al. [2] report that more research is needed to determine which interventions provide the best results in the treatment of chronic neck pain.

A second method for treating chronic neck pain with very good results is acupuncture $[10,16,19]$. Research has shown that acupuncture effectively reduces pain and improves the functional ability of people with chronic neck pain $[10,16,26]$. However, although recent research data suggests acupuncture as an appropriate and effective treatment for chronic pain $[27,28]$, the efficacy of the technique is disputed by various researchers $[26,29]$. The aim of this study is to compare the efficacy of these two techniques through the application of two different protocols that were applied to patients with chronic neck pain.

\section{MATERIALS AND METHODS}

\section{Design}

This was an assessor-blind randomized clinical trial conducted under the supervision of the Department of Physiotherapy of the International Hellenic University during the period November 2020-April 2021. Study was conducted following the Consolidated Standards of Reporting Trials (CONSORT) extension for pragmatic clinical trials. The 45 participants with chronic neck pain were randomly divided into three groups (two intervention and one control) of 15 people each. The distribution of participants was done through the Research Randomizer Computer software (version 4) [30] by an independent researcher. The randomization process was conducted in small groups and thus allocation concealment was achieved. The first intervention group followed a treatment protocol based on the application of joint mobilization (Manual Therapy Group); the second intervention group followed an acupuncture protocol (Acupuncture Group) while the third group (Control) did not follow any treatment. The intervention had a total duration of eight weeks while the frequency of each program was three times a week. This study was performed in compliance with the ethical standards outlined in the Declaration of Helsinki.
Ethical approval was granted by the Ethics Committee of the Department of Physiotherapy of the International Hellenic University University (No. EC-02/2021). Written informed consent was obtained from all subjects in our institution.

\section{Participants}

The inclusion criteria of the participants were: Medical report with a diagnosis of neck pain, referral to a physician for physiotherapy, duration of symptoms longer than three months, and written consent to participate in the research. The exclusion criteria of the participants were: History of acute neck injury and conditions that refer to red flags in neck pain (severe pathology due to vertebral artery syndrome, intramedullary tumor, cervical fractures, cervical vertebral osteomyelitis). The demographic characteristics of the participants in each group are presented in Table 1.

All participants were informed of the purpose of the research and received printed information material for the purposes of the research before signing their participation consent form. To avoid any distortion in the clinical picture of the results, participants were recommended to refrain from the use of painkillers or anti-inflammatory medication for the eight weeks of the program. However, when this was necessary in some cases, we asked the participants to report it to the members of the research team.

\section{Measurements}

The following measurements were performed at the beginning (baseline) and end of the study (eighth week) by the same outcome assessor. All measurements took place in a different room and the assessor was unaware about which group each participant belonged to.

\section{Subjective perception of pain with the visual analog scale (VAS) for pain}

The intensity of the present pain was assessed with the visual analog scale for pain (pain VAS). It is a tool for measuring pain that is essentially based on the subjective selfassessment of the patient. It is a straight $100 \mathrm{~mm}$ horizontal line with the phrase "No Pain" on one side and "Unbearable

Table 1. Demographic characteristics of the participants

\begin{tabular}{cccc}
\hline $\begin{array}{c}\text { Demographic } \\
\text { characteristics }\end{array}$ & $\begin{array}{c}\text { Manual } \\
\text { therapy group }\end{array}$ & $\begin{array}{c}\text { Acupuncture } \\
\text { group }\end{array}$ & $\begin{array}{c}\text { Control } \\
\text { group }\end{array}$ \\
\hline $\begin{array}{c}\text { Number of } \\
\text { participants }\end{array}$ & 15 & 15 & 15 \\
$\begin{array}{c}\text { Age (years) } \\
\text { Gender (\%) } \\
\text { (Women) }\end{array}$ & $71 \pm 7.69$ & $40 \pm 3.93$ & $44 \pm 4.3$ \\
\hline
\end{tabular}


Pain" on the other. The patient is asked to indicate or mark the point of this line that they consider representative of their pain levels. The examiner then measures the distance with a ruler in $\mathrm{mm}$. This scale is widely used in research as a tool for measuring pain and shows satisfactory indices of reliability and validity in people with neck pain $[31,32]$.

\section{Functional ability with the Neck Disability Index (NDI) questionnaire}

The Neck Disability Index (NDI) is a self-report questionnaire for inability to perform daily activities due to neck pain $[33,34]$. It is a widely used tool with a high reliability index $(\mathrm{r}=0.89)$, used in clinical practice and research to assess the degree of disability of the examinee (Vernon and Mior, 1991 [35]). The questionnaire includes 10 questions related to activities regarding personal care, weightlifting, driving, work, sleep, entertainment, and other everyday life skills. Each question can have 6 possible answers, which are scored on a scale of $0-5$. The total score resulting from the sum of all the answers ranges from zero (no disability) to 50 (complete disability) [33].

\section{Experimental protocols}

Each of the two intervention protocols had a session duration of 30 minutes. Both intervention groups completed a total of 24 sessions over eight weeks. An Orthopedic Manual Therapy (OMT) physiotherapist specializing in manual mobilization with eight years of clinical experience applied the protocol to the manual therapy team, while a second physiotherapist with two years of training in acupuncture and seven years of relevant clinical experience applied the treatment protocol to the second intervention group (acupuncture group).

\section{Manual therapy protocol}

Prior to the application of the joint mobilization manipulations, the physiotherapist examined the mobility of the cervical and thoracic vertebrae to locate the areas with impaired movement. This assessment was performed through the Posterior-Anterior Segmental Mobility test $[36,37]$. The aim of this test is to detect pain and impairments in the cervical and thoracic joints by assessing their mobility [36]. The procedure is as follows: (1) The patient is placed in a prone position, (2) The examiner stands directly behind their head, (3) The examiner places their hands (specifically only their thumbs) on the spinous processes of the cervical vertebrae with their elbows extended so as to utilize the motion of their entire upper torso, and finally, (4) The examiner exercises on each vertebra separately a posterior to anterior force in a progressive oscillatory fashion controlling the mobility of each vertebra. The procedure for assessing the thoracic vertebrae is similar; the examiner also exerts a posterior to anterior force in a progressive oscillatory fashion controlling the mobility of each thoracic vertebra. The difference is that the pressure originates from the back of their hands (opisthenar) and not the thumbs. The test is deemed successful when the pressure reproduces the symptoms of the patient. The assessor uses their experience and sensation to categorize each vertebral segment as "normal", "hyperactive" or "hypoactive". Furthermore, the underlying and overlying vertebral sections are used to compare and evaluate the mobility of each examined section [36].

Joint mobilization was then performed to the cervical and thoracic vertebrae with emphasis on the points where the limitation had been identified during the evaluation. Joint mobilization involved pulling and sliding with the patient in supine position and sitting. The protocol we implemented was based on the protocol of Lopez-Lopez et al. [24]. Cervical pulling was applied with the patient lying down and the hands of the physiotherapist were placed on their occipital bone. The physiotherapist was pulling from the occipital bone of the patient using both hand manipulations as well as a stabilization belt, which was passing around the pelvis of the physiotherapist and ending at the dorsal surface of both their hands. The patient was then placed in a sitting position and sliding was performed on the respective vertebral sections. The patient should not feel pain during the manipulations. The procedure to perform joint mobilization on the cervical vertebrae is the following: (1) The patient is in a prone position on the stretcher with both their hands under their forehead, (2) The therapist places both their thumbs on the spinous processes of the C2 vertebra of the patient and (3) The therapist performs grade III posteroanterior impulses with a duration of two minutes and a speed of $2 \mathrm{~Hz}$ (120 movements per minute). This procedure is repeated threefold with oneminute rest periods between each application.

\section{Acupuncture protocol}

Before inserting the special needles, the physiotherapist disinfected the skin with a cotton swab with pure alcohol. $25 \times 30 \mathrm{~mm}$ needles were used for acupuncture (Dongbang DB105 spring handle needle). Two types of acupuncture disposable needles were used in terms of length: 1.0 and 1.5 cun. The 1st ones were inserted into the Houxi (SI3) acupoints and the latter into the Fenhchi (GB20) and Jianliao (TE14) acupoints. Traditional Chinese Medicine (more specifically its meridian theory) states that the central neckline is part of the governor vessel, to which the Houxi acupoints connect. This reason led to the selection of these acupoints. Additionally, needles were inserted into the suboccipital area (BL10, GV16, GB20), and locally into the neck and shoulder area (Jianjing GB21, Jianwaishu SI14, Jianzhongshu (SI15). Moreover, a 
"Qi" sensation was achieved by twisting the needles after their insertion. Lastly, after twisting the needles, they were left undisturbed for 25 minutes. This protocol was based on that of Sun et al. [29]. The duration of the application was 30 minutes.

\section{Sample size calculation}

A total sample size of at least 51 subjects was calculated based on an a priori power analysis ( $G^{*}$ Power 3.0.10). As a basic prerequisite for this calculation, the power $(1-\beta)$ was set at $95 \%$, and the detection of a difference in the order $\mathrm{f}=.5$ (Cohen's f) [38]. The alpha was set at .05 for all tests. Despite strong efforts, the targeted sample size of $\mathrm{n}=51$ was not reached. Due to the difficulties caused by the pandemic, it was not possible to include more than 45 patients in the study.

\section{Statistical analysis}

Data were analyzed using SPSS Statistics for Windows, Version 25.0 (SPSS Inc., Chicago, IL, USA). The ShapiroWilk test and Q-Q and P-P plots were used to check normal distribution. A two-way analysis of variance (ANOVA) with repeated measures was applied. The ANOVA was applied to examine the interaction effect of "Group" and "Time of measurement". The "Group" factor was tested at three levels (Manual therapy group [MT], Acupuncture group [AG] and Control group [CG]), while the factor "Time of measurement" was tested at two levels (before and after the intervention). If the "Group" $\times$ "Time" interaction effects were statistically significant, the simple main effects were reported using Tukey's post-hoc test (HSD). The significance level was set at $p<.05$.

\section{RESULTS}

Between November 2020-December 2020, a total of 63 persons were screened for eligibility. Only 45 of them (71.4\%) were included in the study and randomly allocated into one of the three groups (interventions or control) (Fig. 1). None of the participants quit the research prematurely. All 45 participants completed the eight weeks of intervention. There were no missed sessions or missed measurement appointments during the conduction of research. Also, none of the participants experienced any unwanted side effects during the implementation of the treatment protocols.

\section{Pain VAS score}

The analysis of variance revealed an interaction between the "Group" factor and the "Time of measurement" factor $(p<.001)$, a major effect on the "Time of measurement" factor $(p<.001)$ and a major effect on the "Group" factor $(p<.001)$. The post-hoc test showed that the acupuncture group had a statistically significantly lower value on the pain scale compared to the other two groups $(p<.001)$ after the intervention, while there was a statistically significant improvement between the manual therapy and control groups $(p<.05)$ (Table 2$)$. The results of our study revealed that both intervention groups exhibited reduced levels of pain after the end of the intervention compared to the control group. However, in the acupuncture group the reduction in pain was greater than in the manual therapy group with a
CONSORT 2010 flow diagram

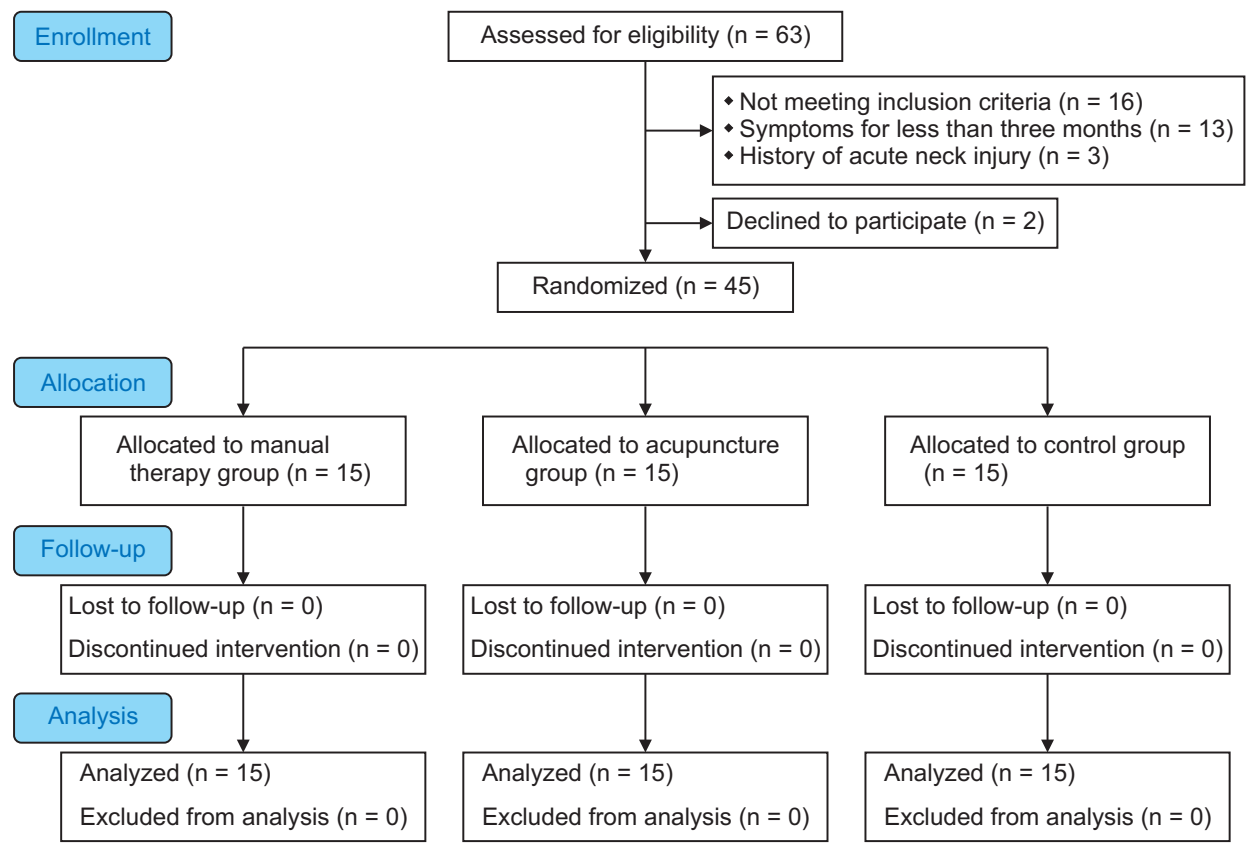

Fig. 1. CONSORT flow diagram of the study. CONSORT = Consolidated Standards of Reporting Trial. 
Table 2. Outcome measures mean scores (SD) and $p$-values of the three groups after intervention

\begin{tabular}{|c|c|c|c|c|}
\hline \multirow{2}{*}{ Outcome measures } & Baseline & 8 weeks & \multirow{2}{*}{$\begin{array}{c}p \text {-value } \\
\text { (between } \\
\text { group) }\end{array}$} & \multirow{2}{*}{$\begin{array}{l}\text { Post hoc Tukey's test } \\
\text { ( } p \text {-value })\end{array}$} \\
\hline & Mean (SD) & Mean (SD) & & \\
\hline \multicolumn{5}{|l|}{ VAS pain score } \\
\hline Acupuncture group $(n=15)$ & $60.21(9.28)$ & $22.25(9.35)$ & $<0.001 *$ & Acupuncture vs control $\left(<0.001^{*}\right)$ \\
\hline Manual Therapy group $(n=15)$ & $59.22(8.64)$ & $31.34(8.78)$ & & Acupuncture vs manual $\left(<0.001^{*}\right)$ \\
\hline Control group $(\mathrm{n}=15)$ & $58.72(10.21)$ & $57.92(10.21)$ & & Manual vs control $\left(0.004^{*}\right)$ \\
\hline \multicolumn{5}{|l|}{ NDI } \\
\hline Acupuncture group $(\mathrm{n}=15)$ & $26.90(4.25)$ & $12.11(6.34)$ & $0.002 *$ & Acupuncture vs control $\left(0.002^{*}\right)$ \\
\hline Manual Therapy group $(n=15)$ & $27.11(5.23)$ & $15.65(5.61)$ & & Acupuncture vs manual $\left(0.007^{*}\right)$ \\
\hline Control group $(n=15)$ & $27.01(4.21)$ & $27.00(6.37)$ & & Manual vs control $\left(0.01^{*}\right)$ \\
\hline
\end{tabular}

VAS $=$ visual analog scale, NDI = neck disability index. $*$ Statistically significant $p$-value.

statistically significant difference $(p<.05)$.

\section{NDI score}

From the analysis of variance, an interaction was found between the "Group" factor and the "Time of measurement" $(p<.05)$, a major effect on the "Time of measurement" factor $(p<.05)$ and a major effect on the "Group" factor $(p<.001)$. The post-hoc test showed that the acupuncture group had a statistically significantly lower value in the NDI score compared to the other two groups $(p<.05)$ after the intervention, while there was a statistically significant improvement between the manual therapy and control groups $(p<.05)$ (Table 2). The results of our study showed that both intervention groups showed reduced levels of disability after the completion of the intervention compared to the control group. However, in the acupuncture group this reduction was greater than in the manual therapy group with a statistically significant difference $(p<.05)$.

\section{DISCUSSION}

The aim of this randomized clinical trial was to compare the efficacy of two different therapeutic approaches, that of joint mobilization and that of acupuncture in pain and disability of patients with chronic neck pain. Although there is evidence that both techniques are effective in improving symptoms in people with chronic neck pain, there is limited evidence as to which of the two methods is more effective. The results of this randomized clinical trial showed that both interventions significantly improved levels of pain and disability compared to control group participants. The findings of our study are in line with those of other studies regarding the efficacy of both joint mobilization $[39,40]$ and acupuncture $[26,41]$ in individuals with chronic neck pain. However, the improvement in pain and disability was greater in the participants of the acupuncture group than in the participants of the manual therapy group, which means that the acupuncture protocol proved more effective than the manual therapy protocol.

This greater reduction in the VAS pain score presented in the acupuncture group compared to the manual therapy group may be due to the mechanism through which acupuncture induces analgesia. Previous research has shown that the analgesic effect of acupuncture may be related to the activation of centrifugal nerve fibers $\mathrm{A} \delta$ and $\mathrm{C}$ in muscles when the needle is inserted at acupuncture points, as the signals are transmitted directly to the spinal cord and through the centrifugal pathways to midbrain $[42,43]$. This analgesic mechanism in the participants of the acupuncture group may have been the reason why the participants in this group experienced lower levels of pain compared to the participants in the manual therapy group. The findings of our study are consistent with those of previous studies that also found the analgesic effect of acupuncture in patients with neck pain $[19,41]$. However, they contrast those of Sun et al. [29], who did not find a significant effect on pain after applying a corresponding acupuncture protocol for three weeks in 34 patients with chronic neck pain. One explanation for this may be the time factor; the possibility that the three-week period was too short for significant differences to occur. In contrast, in other studies in which longer-term acupuncture protocols were applied, a strong analgesic effect with the application of acupuncture to chronic pain was identified [19].

A similar improvement in the participants of the acupuncture group was also found in the NDI score, meaning that the patients of the acupuncture group experienced fewer functional limitations than those of the manual therapy group. This is a logical consequence, since the participants of the acupuncture group experienced lower levels of pain and were therefore expected to show fewer functional limitations compared to the participants of the manual therapy group.

The main limitation of the research was the small number 
of samples, which was caused by the fact that this research was conducted during the COVID-19 pandemic period, when in Greece strict restrictions on the movement of citizens were imposed.

\section{CONCLUSIONS}

Applying an eight-week acupuncture protocol three times a week seemed more effective than applying an equal frequency and duration joint mobilization protocol to patients with chronic neck pain to reduce pain and disability. Both joint mobilization and acupuncture appear to be effective interventions in the treatment of chronic neck pain. However, it seems that the analgesic effect of acupuncture is greater. The further reduction of pain and disability experienced by acupuncture is significant as it is associated with fewer functional limitations, reduced drug administration, and reduced medical care costs for people with chronic neck pain; thus, economically unburdening the health system.

\section{CONFLICT OF INTEREST}

The authors declare no conflict of interest.

\section{ORCID}

Petros Voulgarakis, https://orcid.org/0000-0002-7906-5514

Paris Iakovidis, https://orcid.org/0000-0002-0451-9577

Dimitrios Lytras, https://orcid.org/0000-0001-6562-2036

Ioanna P. Chatziprodromidou, https://orcid.org/0000-0001-6242-1351

Anastasios Kottaras,

https://orcid.org/0000-0002-7035-8927

Thomas Apostolou,

https://orcid.org/0000-0002-5069-8035

\section{REFERENCES}

1. Misailidou V, Malliou P, Beneka A, Karagiannidis A, Godolias G. Assessment of patients with neck pain: a review of definitions, selection criteria, and measurement tools. J Chiropr Med 2010;9:49-59.

2. Tsakitzidis G, Remmen R, Peremans L, Van Royen P, Duchesnes C, Paulus D, et al. Non-specific neck pain: diagnosis and treatment. Available at: https://kce.fgov.be/sites/default/files/ atoms/files/d20091027356.pdf [Date accessed: April 20, 2021]

3. Côté P, Cassidy DJ, Carroll LJ, Kristman V. The annual incidence and course of neck pain in the general population: a population-based cohort study. Pain 2004;112:267-73.

4. Bertozzi L, Gardenghi I, Turoni F, Villafañe JH, Capra F, Guccione AA, et al. Effect of therapeutic exercise on pain and disability in the management of chronic nonspecific neck pain: systematic review and meta-analysis of randomized trials. Phys Ther 2013;93:1026-36.

5. Guzman J, Hurwitz EL, Carroll LJ, Haldeman S, Côté P, Carragee EJ, et al. A new conceptual model of neck pain: linking onset, course, and care: the Bone and Joint Decade 2000-2010 Task Force on Neck Pain and Its Associated Disorders. Spine (Phila Pa 1976) 2008;33(4 Suppl):S14-23.

6. Martin BI, Deyo RA, Mirza SK, Turner JA, Comstock BA, Hollingworth W, et al Expenditures and health status among adults with back and neck problems. JAMA 2008;299:656-64.

7. Manchikanti L, Singh V, Datta S, Cohen SP, Hirsch JA. Comprehensive review of epidemiology, scope, and impact of spinal pain. Pain Physician 2009;12:E35-70.

8. Cohen SP. Epidemiology, diagnosis, and treatment of neck pain. Mayo Clin Proc 2015;90:284-99.

9. Gross AR, Paquin JP, Dupont G, Blanchette S, Lalonde P, Cristie T, et al. Exercises for mechanical neck disorders: a Cochrane review update. Man Ther 2016;24:25-45.

10. Witt CM, Jena S, Brinkhaus B, Liecker B, Wegscheider K, Willich SN. Acupuncture for patients with chronic neck pain. Pain 2006;125:98-106.

11. Yang Y, Yan X, Deng H, Zeng D, Huang J, Fu W, et al. The efficacy of traditional acupuncture on patients with chronic neck pain: study protocol of a randomized controlled trial. Trials 2017;18:312.

12. Childs JD, Cleland JA, Elliott JM, Teyhen DS, Wainner RS, Whitman JM, et al. Neck pain: clinical practice guidelines linked to the International Classification of Functioning, Disability, and Health from the Orthopedic Section of the American Physical Therapy Association. J Orthop Sports Phys Ther 2008;38:A1-34.

13. Liu R, Kurihara C, Tsai HT, Silvestri PJ, Bennett MI, Pasquina PF, et al. Classification and treatment of chronic neck pain: a longitudinal cohort study. Reg Anesth Pain Med 2017;42:52-61.

14. Bernal-Utrera C, Gonzalez-Gerez JJ, Anarte-Lazo E, RodriguezBlanco C. Manual therapy versus therapeutic exercise in nonspecific chronic neck pain: a randomized controlled trial. Trials 2020;21:682.

15. Miller J, Gross A, D'Sylva J, Burnie SJ, Goldsmith CH, Graham $\mathrm{N}$, et al. Manual therapy and exercise for neck pain: a systematic review. Man Ther 2010;15:334-54.

16. Blossfeldt P. Acupuncture for chronic neck pain--a cohort study in an NHS pain clinic. Acupunct Med 2004;22:146-51.

17. Lytras DE, Sykaras EI, Christoulas KI, Myrogiannis IS, Kellis E. Effects of exercise and an integrated neuromuscular inhibition technique program in the management of chronic mechanical neck pain: a randomized controlled trial. J Manipulative Physiol Ther 2020;43:100-13.

18. Lytras D, Sykaras E, Christoulas K, Myrogiannis I, Kellis E. Effects of an integrated neuromuscular inhibition technique 
program on neck muscle strength and endurance in individuals with chronic mechanical neck pain. J Bodyw Mov Ther 2019;23: 643-51.

19. McKee MD, Kligler B, Fletcher J, Biryukov F, Casalaina W, Anderson B, et al. Outcomes of acupuncture for chronic pain in urban primary care. J Am Board Fam Med 2013;26:692-700.

20. Rushton A, Beeton K, Jordaan R, Langendoen J, Levesque L, Maffey L, et al. Educational Standards in Orthopaedic Manipulative Therapy. Albany: International Federation of Orthopaedic Manipulative Physical Therapists (IFOMPT), 2016.

21. Puniello MS, Courtney C, Devaney L, King PM, Kring R, Lonnemann E, et al. Orthopaedic Manual Physical Therapy. Description of Advanced Specialty Practice. Baton Rouge: American Academy of Orthopaedic Manual Physical Therapists, 2018.

22. Blanpied PR, Gross AR, Elliott JM, Devaney LL, Clewley D, Walton DM, et al. Neck pain: revision 2017. J Orthop Sports Phys Ther 2017;47:A1-83.

23. Häkkinen A, Salo P, Tarvainen U, Wirén K, Ylinen J. Effect of manual therapy and stretching on neck muscle strength and mobility in chronic neck pain. J Rehabil Med 2007;39:575-9.

24. Lopez-Lopez A, Alonso Perez JL, González Gutierez JL, La Touche R, Lerma Lara S, Izquierdo H, et al. Mobilization versus manipulations versus sustain apophyseal natural glide techniques and interaction with psychological factors for patients with chronic neck pain: randomized controlled trial. Eur J Phys Rehabil Med 2015;51:121-32.

25. Beltran-Alacreu H, López-de-Uralde-Villanueva I, FernándezCarnero J, La Touche R. Manual therapy, therapeutic patient education, and therapeutic exercise, an effective multimodal treatment of nonspecific chronic neck pain: a randomized controlled trial. Am J Phys Med Rehabil 2015;94(10 Suppl 1):887-97.

26. Fu LM, Li JT, Wu WS. Randomized controlled trials of acupuncture for neck pain: systematic review and meta-analysis. J Altern Complement Med 2009;15:133-45.

27. Vickers AJ, Linde K. Acupuncture for chronic pain. JAMA 2014;311:955-6.

28. Vickers AJ, Cronin AM, Maschino AC, Lewith G, MacPherson $\mathrm{H}$, Foster NE, et al. Acupuncture for chronic pain: individual patient data meta-analysis. Arch Intern Med 2012;172:1444-53.

29. Sun MY, Hsieh CL, Cheng YY, Hung HC, Li TC, Yen SM, et al. The therapeutic effects of acupuncture on patients with chronic neck myofascial pain syndrome: a single-blind randomized controlled trial. Am J Chin Med 2010;38:849-59.

30. Urbaniak GC, Plous S. Research randomizer (version 4.0). Available at: https://www.randomizer.org/ [Date accessed: August 19, 2021]

31. Hawker GA, Mian S, Kendzerska T, French M. Measures of adult pain: Visual Analog Scale for Pain (VAS Pain), Numeric
Rating Scale for Pain (NRS Pain), McGill Pain Questionnaire (MPQ), Short-Form McGill Pain Questionnaire (SF-MPQ), Chronic Pain Grade Scale (CPGS), Short Form-36 Bodily Pain Scale (SF-36 BPS), and Measure of Intermittent and Constant Osteoarthritis Pain (ICOAP). Arthritis Care Res (Hoboken) 2011;63 Suppl 11:S240-52.

32. Clark P, Lavielle P, Duarte C. Patient ratings of care at a rheumatology out-patient unit. Arch Med Res 2004;35:82-6.

33. Vernon H. The Neck Disability Index: state-of-the-art, 19912008. J Manipulative Physiol Ther 2008;31:491-502.

34. Trouli MN, Vernon HT, Kakavelakis KN, Antonopoulou MD, Paganas AN, Lionis CD. Translation of the Neck Disability Index and validation of the Greek version in a sample of neck pain patients. BMC Musculoskelet Disord 2008;9:106.

35. Vernon H, Mior S. The Neck Disability Index: a study of reliability and validity. J Manipulative Physiol Ther 1991;14:40915.

36. Rey-Eiriz G, Alburquerque-Sendín F, Barrera-Mellado I, Martín-Vallejo FJ, Fernández-de-las-Peñas C. Validity of the posterior-anterior middle cervical spine gliding test for the examination of intervertebral joint hypomobility in mechanical neck pain. J Manipulative Physiol Ther 2010;33:279-85.

37. Cleland JA, Childs JD, Fritz JM, Whitman JM. Interrater reliability of the history and physical examination in patients with mechanical neck pain. Arch Phys Med Rehabil 2006;87: 1388-95.

38. Cohen L, Manion L, Morrison K. Research Methods in Education, 6th ed. New York: Routledge, 2007. 638 p.

39. Coulter ID, Crawford C, Vernon H, Hurwitz EL, Khorsan R, Booth MS, et al. Manipulation and mobilization for treating chronic nonspecific neck pain: a systematic review and metaanalysis for an appropriateness panel. Pain Physician 2019;22: E55-70.

40. Vernon H, Humphreys K, Hagino C. Chronic mechanical neck pain in adults treated by manual therapy: a systematic review of change scores in randomized clinical trials. J Manipulative Physiol Ther 2007;30:215-27.

41. Vas J, Perea-Milla E, Méndez C, Sánchez Navarro C, León Rubio JM, Brioso M, et al. Efficacy and safety of acupuncture for chronic uncomplicated neck pain: a randomised controlled study. Pain 2006;126:245-55.

42. Kawakita K, Okada K. Acupuncture therapy: mechanism of action, efficacy, and safety: a potential intervention for psychogenic disorders? Biopsychosoc Med 2014;8:4.

43. Li X, Wang R, Xing X, Shi X, Tian J, Zhang J, et al. Acupuncture for myofascial pain syndrome: a network meta-analysis of 33 randomized controlled trials. Pain Physician 2017;20:E883-902. 